\title{
Sex-specific spatio-temporal variability in reproductive success promotes the evolution of sex-biased dispersal
}

\author{
Andreas Gros ${ }^{\text {a,* }}$ Hans Joachim Poethke ${ }^{\text {a,1 }}$ \\ Thomas Hovestadt ${ }^{\mathrm{a}, 2}$ \\ a University of Würzburg, Field Station Fabrikschleichach, Glashüttenstrasse 5, \\ D-96181 Rauhenebrach, Germany
}

Postprint of article published in Theoretical Population Biology 76 (2009) 13-18 http://dx.doi.org/10.1016/j.tpb.2009.03.002 


\begin{abstract}
Inbreeding depression, asymmetries in costs or benefits of dispersal, and the mating system have been identified as potential factors underlying the evolution of sexbiased dispersal. We use individual-based simulations to explore how the mating system and demographic stochasticity influence the evolution of sex-specific dispersal in a metapopulation with females competing over breeding sites, and males over mating opportunities. Comparison of simulation results for random mating with those for a harem system (locally, a single male sires all offspring) reveal that even extreme variance in local male reproductive success (extreme male competition) does not induce male-biased dispersal. The latter evolves if between-patch variance in reproductive success is larger for males than females. This can emerge due to demographic stochasticity if habitat patches are small. More generally, members of a group of individuals experiencing higher spatio-temporal variance in fitness expectations may evolve to disperse with greater probability than others.
\end{abstract}

Key words: sex-biased dispersal, demographic stochasticity, metapopulation, individual-based simulation, sex-specific competition

\footnotetext{
* andigros72@googlemail.com

1 poethke@biozentrum.uni-wuerzburg.de

2 hovestadt@biozentrum.uni-wuerzburg.de
} 


\section{Introduction}

Dispersal is an ecological key mechanism with far reaching consequences for populations and communities (e.g. Clobert et al., 2001; Chave, 2004; Hanski and Gaggiotti, 2004). Just as important, dispersal "decisions" have a profound influence on an individual's (inclusive) fitness. Empirical evidence as well as theory thus indicates that dispersal decisions should often be conditiondependent (e.g. Bowler and Benton, 2005), as the fitness consequences of such decisions depend on external as well as the internal conditions of an individual (Clobert et al., 2009).

Sex-biased dispersal is a prominent example of condition-dependent dispersal observed in many taxa (Greenwood, 1980; Greenwood and Harvey, 1982; Liberg and von Schantz, 1985; Pusey, 1987; Baguette et al., 1998; Sundström et al., 2003; Möller and Beheregaray, 2004; Williams and Rabenold, 2005; Hammond et al., 2006; Lawson Handley and Perrin, 2007; Öckinger and Smith, 2007). The conspicuousness and relevance of this phenomenon has also attracted the attention of theoreticians who wish to understand, under which conditions sex-biased dispersal is likely to evolve. Avoidance of inbreeding depression has been identified as an especially likely factor promoting the evolution of sex-biased dispersal; under many conditions inbreeding could even lead to a situation where only one sex disperses while the other remains completely philopatric (Gandon, 1999; Perrin and Mazalov, 1999, 2000). This asymmetry should be more pronounced in polygynous mating system than under monogamy as the likelihood of mating with close relatives is higher in the former (Perrin and Mazalov, 1999).

More importantly, inbreeding avoidance as such cannot predict which of the two sexes should disperse - this would completely depend on historical chance events (Perrin and Mazalov, 1999; Gandon, 1999; Gros et al., 2008). Thus, additional explanations for the evolution of sex-biased dispersal have been suggested, which either on their own or in conjunction with the influence of inbreeding can explain which of the two sexes disperses more. One obvious possibility is that dispersal is more costly for one than the other sex. In that case the gender with lower costs is likely to become the more dispersive sex, even though it is not completely impossible that the sex with higher costs evolves to disperses more (Perrin and Mazalov, 1999; Wild and Taylor, 2004; Leturque and Rousset, 2004; Gros et al., 2008).

Other explanations assume that the benefits of dispersal and philopatry may not be identical in the two sexes. Greenwood $(1980,1983)$ has, for example, suggested that for male birds the benefits from taking over the natal territory are larger than they are for females; consequently females are more likely to emigrate than males in many bird species (Greenwood and Harvey, 1982). Hirota $(2004,2005)$ has predicted the evolution of female biased dispersal under the premise that females mate before emigration and that empty habitats are available in the landscape. In this case, females can harvest great fitness benefits from colonizing such empty habitat patches while males could not 
do so; and as the females are already fertilized they transport not only their own genes but also the genes of their mating partner(s). It should be noted, however, that mating before emigration is not likely to occur if avoidance of inbreeding is a strong driver of dispersal.

Perrin and Mazalov (2000) have drawn attention to the fact that the intensity of competition may be different for the two sexes as males and females potentially compete over different resources, e.g. females over food and males over mating opportunities. The asymmetry should be weakest in monogamous systems and strongest in systems where few males manage to monopolize access to many males, e.g. harem- or lek-systems. Birds, for example, are mostly monogamous and share the raising of offspring - and dispersal is usually female-biased, which is often attributed to the benefit of philopatry for keeping a territory in males (Greenwood, 1980; Clarke et al., 1997). Mammals, on the other hand are often polygynous and dispersal is usually male-biased (Lawson Handley and Perrin, 2007). More recently, several publications report male-biased dispersal in various fishes (Anseeuw et al., 2008; Cano et al., 2008) and reptiles (Keogh et al., 2007; Dubey et al., 2008; Ujvari et al., 2008) with polygynous mating systems, and sex-biased dispersal is also known in insects, e.g. Baguette et al. (1998); Beirinckx et al. (2006). However, exceptions to the general picture exists, and generally, the link between sex-biased dispersal and mating system may be confounded by the link with the social system and kin-cooperation (Lawson Handley and Perrin, 2007).

In their analytical model, Perrin and Mazalov (2000) showed that the mating system could promote the evolution of sex-biased dispersal, even in the absence of inbreeding depression. However, according to their model male-biased dispersal emerges only if resource competition among females (in the breeding areas) is completely removed, i.e. if populations are allowed to grow exponentially. Otherwise, they always predict the evolution of balanced dispersal. The assumption of exponential growth is, however, rather unrealistic, especially for birds and mammals, which nonetheless very often show sex-biased dispersal (Dobson, 1982; Greenwood and Harvey, 1982).

Perrin and Mazalov (2000) could - because they used a deterministic model without demographic and/or environmental stochasticity - not recognize another mechanism by which the mating system may influence the evolution of sex-biased dispersal. In this paper we will show that - even in the absence of inbreeding depression - differences in the between-patch variability in reproductive success promotes sex-biased dispersal. Both, within- and between-patch variability in reproductive success only occurs in stochastic population models; and the magnitude of this variability is, at least partly, an emergent consequence of dispersal (cf. Poethke et al., 2003; Ronce, 2007). These boundary conditions foreclose a (comprehensible) analytical approach to study the influence of variability on the evolution of evolutionary stable dispersal strategies in stochastic metapopulation models. We thus use an individual-based model to investigate the effects of kin-competition, demographic variability, and the mating system on the evolution of sex-biased dispersal. However, instead of 
eliminating resource-competition among females as Perrin and Mazalov (2000) did, we intensify mate-competition among males by introducing a harem mating system in which a single male fertilizes all females in a patch.

\section{The model}

To investigate the combined influence of mate- and resource-competition on the evolution of gender-specific dispersal, we modify an individual-based model more completely described in Poethke and Hovestadt (2002). The assumption we implement in the model are principally identical to those of Perrin and Mazalov (2000): we simulate the population dynamics, inter-patch dispersal, and the evolution of emigration probability in a metapopulation of a diploid and sexually reproducing annual species with discrete generations. Each habitat patch $\left(p_{i}\right)$ supports maximally $K$ breeding females (territories). Simulation experiments are initialised by placing $N=2 K$ individuals with a sex-ratio of one in each patch. As stochastic demographic effects become less prominent with increasing population size, we use different carrying capacities $(K)$ to investigate the influence of demographic stochasticity on the evolutionary outcome. To keep the number of individuals (and thus of mutation events) in the whole metapopulation comparable (approximately 25.000 reproducing individuals) we increase the number of local populations in correspondence with the reduction in $K$. Thus, the meta-population consists of 256, 676 and 1296 habitat patches with a local carrying capacity $(K)$ of 50,20 , and 10 territories, respectively. Additional test runs clearly indicate that in the relevant parameter range patch number did not have an effect on simulation results. Each mated female produces an equal number of offspring $(\lambda)$ with a standard value of $\lambda=3$. Yet, to explore the importance of the magnitude of $\lambda$ we also conducted tests with $\lambda=6$. All offspring mature and can disperse once before maturation. If individuals disperse, they die during transition with probability $c \in\{0.05,0.40\}$. Like Perrin and Mazalov (1999) we assume global dispersal, i.e. a surviving disperser arrives in any other patch with equal probability. After the dispersal phase females compete for the breeding spaces (territories) and males for mating with the successful females. Both, the females successfully occupying territories and their mating partners are selected randomly among the females and males respectively present in the patch. In all scenarios we assume that breeding success is not affected by inbreeding.

Each individual is characterised by four alleles $\left(m_{1}, m_{2}, f_{1}, f_{2}\right)$ at two diploid loci $(m, f)$, inherited from its parents. The first locus $(m)$ determines the male's probability for emigration $\left(p_{m}\right)$, the second $(f)$ determines $p_{f}$ in females; for both sexes the phenotype is determined as the mean of the two corresponding parental alleles. In contrast to the setting used in Poethke and Hovestadt (2002) we thus allow for the independent evolution of emigration probabilities in males and females but do not allow for density-dependent emigration. The alleles of these loci can take continuous values between zero and one $\left(m_{i}, f_{i} \in[0,1]\right)$. To test whether an initial bias in dispersal has an effect of the 
outcome of evolution, we initialize simulations for each parameter setting $(\lambda$, $c$, and $K$ ) with 10 different start values for male and female dispersal, always with a population mean emigration probability of $0.2: f_{i}$ is set to a value from $0.02,0.04,0.06 \ldots 0.20$, and the alleles for male emigration probability correspondingly to $m_{i}=0.2-f_{i}$. When inheriting a parents's genes, each allele transferred to the offspring mutates with probability 0.002. If mutations occur, the alleles of the loci $m$ and $f$ are altered by adding a random value drawn from a uniform distribution within the interval [-0.02, 0.02].

We run 50 simulation experiments for each combination of $K, \lambda, c$, and initial emigration probabilities, i.e. $50 \times 3 \times 2 \times 2 \times 10=6000$ simulation runs for each mating system. To allow the populations to reach evolutionary equilibrium, we let simulations run for 50.000 generations. Preliminary runs for various parameter values showed that a stable allele distribution, i.e. evolutionary equilibrium, was usually reached within 20.000 generations, at most after 40.000 generations, when the rate of change per 100 generations in the evolving traits drops from initially about 0.20 (directional) to fluctuating values around zero between -0.04 and 0.04 . We record the resulting mean values for the loci $m$ and $f$, as well as all other measures mentioned below in the gamete phase in the last generation.

We implement two different mating systems to test whether differences in the intensity of competition cause sex-biased dispersal. In our first scenario, "random mating" (RM), all females winning a breeding-space choose a single mate randomly among all males in their patch - the number of mating successes for males is thus a Poisson distributed number with an approximate mean of one. In our second scenario, "harem" $(\mathrm{H})$, a single randomly chosen male mates with all successful females in the patch. Scenario RM is identical with the first scenario in Perrin and Mazalov (2000). However, with scenario H we intensify mate competition among males instead of removing local resource competition among females as Perrin and Mazalov (2000) did in their second scenario. We want to point out that kin-competition is, like in any individual-based simulation, implicitly accounted for in our simulations (Poethke et al., 2007). For the case of strict monogamy, neither our, nor the model by Perrin and Mazalov (2000) would predict the emergence of male-biased dispersal, as males do not underlie any additional variance in reproductive success compared to females. As expected, additional simulations performed for the smallest patch size $(K=10)$, which give the most extreme bias with the other mating systems, did not result in any dispersal bias if we implemented monogamy.

\section{Results}

Not surprisingly, the costs of dispersal have a great influence on the evolving mean emigration probabilities (e.g. Gandon and Rousset, 1999; Gandon, 1999; Perrin and Mazalov, 1999) with much higher dispersal probabilities evolving in the harem mating system $(\mathrm{H})$ compared to the random mating system (RM; 
Figures 1a,b). More importantly, an increase in patch capacity leads to a decline in emigration probability in the RM scenario, while $K$ hardly has an effect on the emigration probability in the $\mathrm{H}$ scenario. In the latter scenario there is a slight increase in mean dispersal propensity over $K$ because of the lower overall competition emerging in the simulations with small patches. Under that condition the available breeding territories are more often not completely occupied and therefore on average less offspring is produced than the maximum possible as all reproducing females get an equal number of offspring. This reduced competition reduces the incentive for dispersal. The weak response to $K$ in the mating system $\mathrm{H}$ can be traced to the fact that $K$ has little influence on the emergence of a kin-structure in the harem mating system where only a single male mates with all females in a patch. In this case, effective population size $N_{e}=4 N_{m} N_{f} /\left(2 N_{m}+N_{f}\right)$ (Wright, 1931; Nomura, 2005) becomes nearly independent of total population size, respectively habitat capacity and cannot become larger than four. With a single mating male $\left(N_{m}=1\right), N_{e}$ equals 3.33, 3.63 , and 3.84 for $K=10,20$ and 50 respectively.

Any bias in the emigration probabilities we observe is in favour of malebiased emigration (Figures 1c, 1d). Its direction and magnitude is not affected by the direction or magnitude of the sex-bias in emigration at initialization, i.e. would not be dominated by chance or historical effects. It is especially noticeable that, in contrast to the prediction of Perrin and Mazalov (2000), malebiased dispersal also evolves in populations with mating-system RM. However, the bias evolving with RM is usually smaller than with mating system H. Note however, that with very high mean dispersal the potential for a sex-bias becomes limited: With small patches male emigration probability should not evolve to such levels that frequently all half-brothers would emigrate. This would not only waste an easy mating opportunity for the native males but also expose their sisters to the risk of not finding a mate at all. Consequently, only the lower mean dispersal propensity with higher dispersal costs $c$ allows for the evolution of a strong bias in dispersal in simulations with small patch sizes in mating system H. Furthermore, any bias decreases with an increasing number of breeding territories for scenario RM (Fig. 1c, d).

According to the arguments put forward by Perrin and Mazalov (2000), the magnitude of the bias should correlate with the discrepancy between the intensity of local resource competition (for females) and local mate competition (for males). As competition about reproductive resources or mating opportunities increases the variance in reproductive output, variance in offspring number is a good measure for the intensity of competition a gender experiences. If we calculate the within-patch variance in reproductive performance before dispersal (Figures 2a, b) we actually find that the argument by Perrin and Mazalov (2000) cannot explain the outcome of our simulations. Neither the variance in reproductive success for each sex, nor the difference in this variance is affected by habitat capacity in the RM scenario. And in the $\mathrm{H}$ scenario the intensity of local mate competition even increases with $K$ (Figures 2a,b). Yet, as already stated, in both scenarios the sex-bias in emigration probability declines with increasing $K$. 

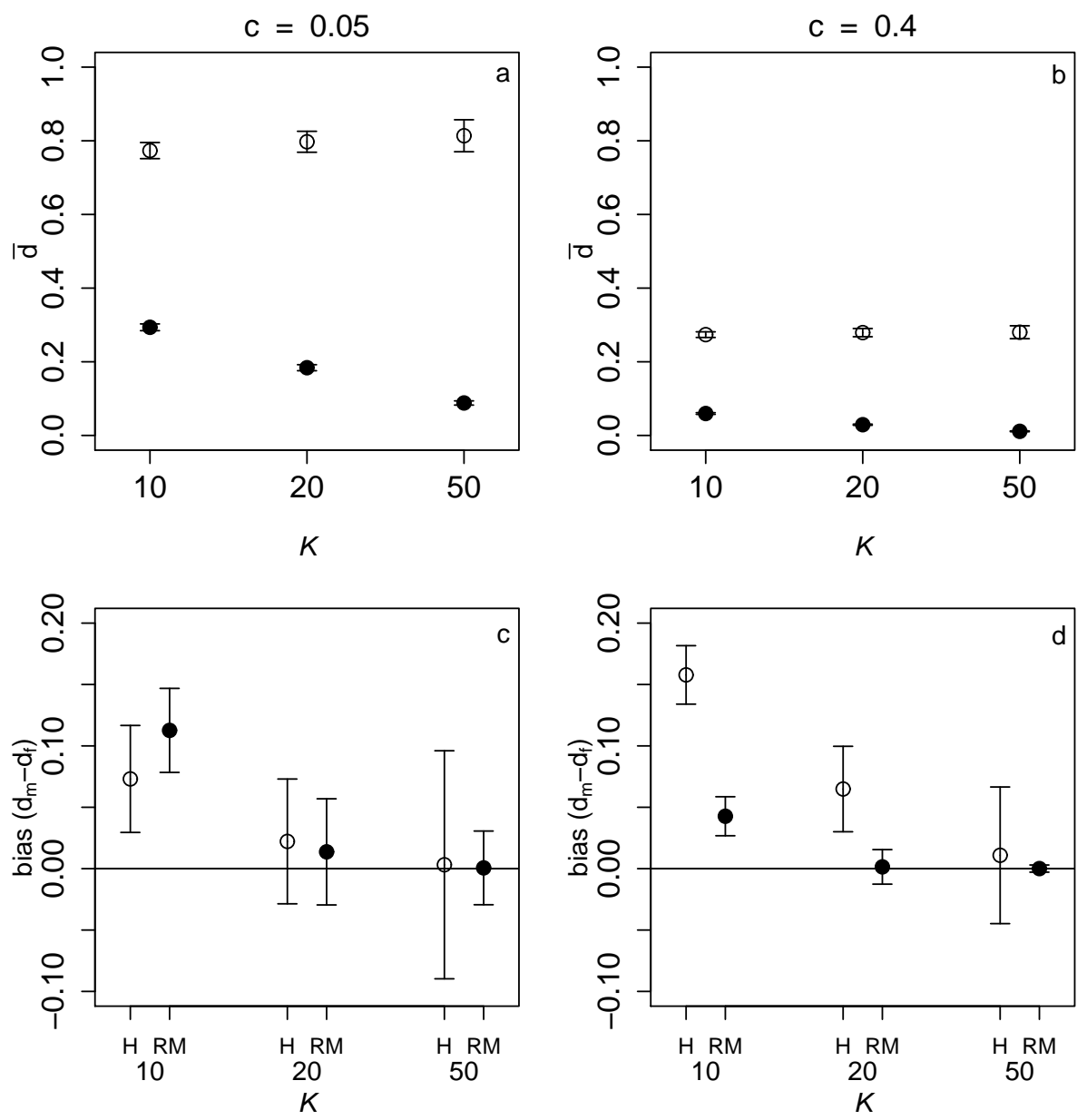

Fig. 1. Top row: Mean emigration probability $\bar{d}$ (averaged of males and females) plotted over habitat capacity ( $K$ ) for mating systems RM (filled symbols) and $\mathrm{H}$ (open symbols). Bottom row: Emerging difference (bias) in mean emigration probability between males and females $\left(d_{m}-d_{f}\right)$ for the same scenarios. Costs of dispersal are low $(c=0.05)$ in the left column $(\mathrm{a}, \mathrm{c})$ and high $(c=0.4)$ in the right column $(\mathrm{b}, \mathrm{d})$. Error bars indicate standard errors in the mean values in the 500 replicate simulation runs.

The driving mechanism behind the selection for male-biased dispersal is, instead, the between-patch variance in reproductive success. Actually, the between-patch variance in local resource and local mate competition becomes virtually identical for large $K$ (Figures 2c, d) in both, the RM and the $\mathrm{H}$ mating scenario. A comparison of Figures $1 \mathrm{c}, \mathrm{d}$ and $2 \mathrm{c}, \mathrm{d}$ makes immediately clear that the male-bias in emigration probability vanishes as soon as there is no more a difference in between-patch variance of reproductive success for females and males. This conclusion is supported by the fact that an increase in fertility from $\lambda=3$ to $\lambda=6$ increases within-patch variability in reproductive success but greatly reduces between-patch variability in reproductive performance of males because then almost all patches are (virtually) filled to carrying capacity as more females compete for them and the number of breeding females becomes almost constant across patches. Consequently, for $\lambda=6$ we hardly see the evolution of sex-biased dispersal, even for the smallest $K$ value (results not shown). 

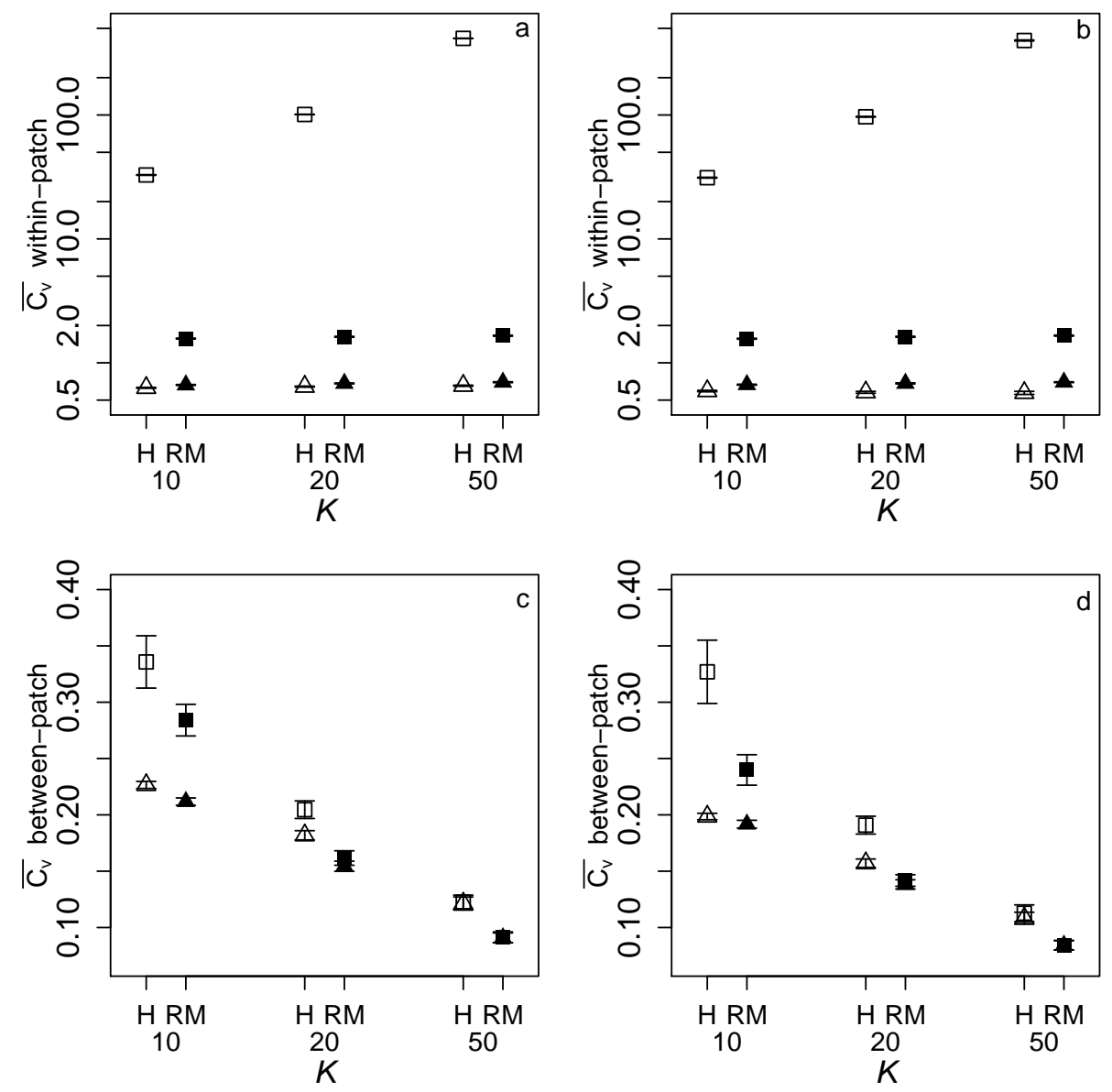

Fig. 2. Coefficient of variation $\left(C_{v} \pm\right.$ s.e. $)$ in reproductive success for females (triangles) and males (squares) in dependence of the mating regime (RM: filled symbols, H: open symbols) and patch capacity $K$. Results are for the same scenarios as those presented in Figure 1. The top-row $(\mathrm{a}, \mathrm{b})$ shows the $C_{v}$ for within-patch variability in per capita reproductive success. The coefficient of variation for the number of offspring was estimated separately for males and females and each patch, and then averaged across patches. In the figures (a,b), mean (across 500 simulation replicates) and standard errors of these means are given (note logarithmic scale of y-axis). The bottom row $(\mathrm{c}, \mathrm{d})$ shows the mean and standard error of the between-patch coefficient of variation in mean per capita reproductive success, separately for males and females. Data presented are mean and standard error from 500 simulations.

\section{Discussion}

The deterministic model of Perrin and Mazalov (2000) predicts that sexbiased dispersal would only evolve if females do not compete among each other at all. In such a case females cannot improve their (inclusive) fitness expectations by dispersing and consequently should avoid the risks of dispersal. However, in our simulations we find that even in the scenario where both, females and males compete (RM) male-biased dispersal evolves as long as patch capacity is small. We further find a difference in sex-bias between the RM and the $\mathrm{H}$ scenario, while, as shown by simple algebraic operations (see Appendix), this 
change in mating system in Perrin and Mazalov's model should not affect the magnitude of sex-bias. Our results cannot be explained by the deterministic model of Perrin and Mazalov (2000). One may deduce from their article, that the mere presence of different intensity in competition is as such not sufficient to promote (male-)biased dispersal. It is important to recognize that Perrin and Mazalov's model predicts male-biased dispersal for a scenario where female reproductive success is not density regulated at all. However, this removes any incentive for dispersal in females, even the effect of kin-competition. Consequently, selection would strongly act against dispersal in females even if the costs of dispersal were extremely small.

Therefore, we have to turn to an alternative explanation for the evolution of sex-biased dispersal emerging in our simulations. It has long been stated that spatio-temporal heterogeneity in reproductive success is, apart from the influence of kin-competition and inbreeding depression, the third principal driver behind the evolution of dispersal (Comins et al., 1980; McPeek and Holt, 1992; Wiener and Tuljapurkar, 1994; Hastings, 1983; Metz and Gyllenberg, 2001). Only if such variability exists can an emigrating individual expect to potentially find another patch where competition is less intense or conditions are in another way more favorable than in its natal patch; this is actually the underlying idea of the "ideal-free distribution" (Fretwell and Lucas Jr., 1970; Cressman and Krivan, 2006). Even though density-dependent emigration would clearly be a better strategy because it uses more information (Clobert et al., 2009), the principle also applies if emigration is unconditional: On average more individuals will (by definition) be located in high-density populations and even unconditional emigration tends to average that out. Our simulations show, that sex-biased dispersal can evolve when the spatio-temporal variability in competition becomes different for the two sexes. This may happen, as soon as fitness expectations for the two sexes depend on different processes. This is the case in our simulations, just as in the model of Perrin and Mazalov (2000), as females compete over a limited number of breeding territories, while the males compete over access to females.

Between-patch variability in resource competition emerges because of demographic stochasticity which leads to variance in female population size. Male reproductive success is affected by this competition too, because it determines the number of mating opportunities in a patch. However, for males the reproductive success also depends on the sex-ratio (in the RM scenario), or the number of competing males (in the $\mathrm{H}$ scenario). Both of these values also underlie demographic variability, especially if $K$ is small. The overall between-patch variability is consequently larger for males than females and we thus observe male-biased emigration in the RM scenario for which the deterministic model of Perrin and Mazalov (2000) predicts symmetric emigration.

It is important to recognize that the difference in within-patch variability of reproductive success in scenarios $\mathrm{RM}$ and $\mathrm{H}$ has, as such, no influence on the sex-bias of dispersal. The larger within-patch variance in the $\mathrm{H}$ scenario only leads to the evolution of higher mean emigration probability because the 
effective population size is (much) smaller than in the RM scenario and kincompetition thus becomes stronger. By dispersing, however, individuals can only exploit the between-patch variance in reproductive success and increase their chances to end in a patch where they have to compete with fewer (closely related) sex-mates over the relevant resources. The male-bias in the latter type of variance declines as habitat capacity increases and consequently dispersal becomes more symmetric with increasing habitat capacity. This may, however, not be the case if apart from demographic stochasticity external (environmental) stochasticity would maintain a sex-bias in between-patch variability even for large habitat patches.

Previous explanations for the evolution of sex-biased dispersal have been based on a number of fundamental principles. First, the avoidance of inbreeding depression, which as such does not, however, predict which of the two sexes should be the more dispersive (e.g. Gandon, 1999; Perrin and Mazalov, 2000). Second, there may exist fundamental asymmetries between the two sexes. These may emerge, for example, because one sex can disperse at a lower cost than the other (e.g. Taylor, 1988; Leturque and Rousset, 2003; Wild and Taylor, 2004; Gros et al., 2008), or because one sex can systematically expect higher fitness benefits when either staying at home (e.g. Greenwood, 1980) or when immigrating into another patch (Hirota, 2004). Hirota (2004) has also pointed out that male genes can "hitchhike" with dispersing females while the alternative option only rarely exists.

Our simulations suggest that there may be a third fundamental principle that can lead to the evolution of sex-biased dispersal: the existence of differences in the sex-specific spatio-temporal variance of fitness expectations in a landscape. Such differences could emerge as soon as the fitness of the two sexes are defined by different processes, which in turn are differently affected by demographic and/or environmental stochasticity. We actually believe that this is not rare and may also apply to individuals which can be classified according to other criteria than gender, e.g. males following different mating strategies than other males. In this case we would expect that it is always the group of individuals, which experiences a larger spatio-temporal variance in fitness expectations that evolves into the more dispersive group.

Acknowledgements - We thank Pleuni Pennings and three anonymous reviewers for their helpful comments on the manuscipt and are grateful for the financial support by the German Science Foundation (DFG PO 244/3-1).

\section{A Appendix}

Perrin and Mazalov (2000) formulate the total fitness of a female as:

$$
W_{x}=x_{i j} \tilde{c} \frac{N b_{0}}{N b(\tilde{x}+x \tilde{c})}+\tilde{x}_{i j} \frac{N\left(k_{y} b_{t}+\tilde{k}_{y} b_{0}\right)}{N b\left(\tilde{x}_{j}+x \tilde{c}\right)}
$$


and, mutatis mutandis, for males total fitness is defined as:

$$
W_{y}=y_{i j} \tilde{c} \frac{N b_{0}}{N b(\tilde{y}+y \tilde{c})}+\tilde{y}_{i j} \frac{N\left(k_{x} b_{t}+\tilde{k}_{x} b_{0}\right)}{N b\left(\tilde{y}_{j}+y \tilde{c}\right)}
$$

$x_{i j}$ is the individual (female) dispersal propensity of individual $i$ in patch $j$, $x_{j}$ denotes the average female dispersal propensity in patch $j$, and $x$ describes the overall mean dispersal propensity for females. $y$ with corresponding subscripts denote the respective male dispersal propensities. $c$ is dispersal mortality, while the ${ }^{\sim}$-sign denotes the complement (e.g. $\left.\tilde{c}=1-c\right) \cdot k_{y}=\tilde{y}_{j} /\left(\tilde{y}_{j}+\tilde{c} y\right)$ is the probability of a male to be philopatric.

For males the first term of the right-hand side of Equation A.2 means that if a male disperses and survives, it competes with $N b(\tilde{y}+y \tilde{c})$ other males for access to $N$ females and will sire $b_{0}$ offspring per successful mating. The second term on the right-hand side of Equation A.2 means that if the male in focus remains philopatric it competes also with $N b\left(\tilde{y}_{j}+y \tilde{c}\right)$ other males (among which $N b \tilde{y}_{j}$ have expected relatedness $r$ ) for access to $N$ females, among which will be

$k_{x}$ immigrants and $\tilde{k}_{x}$ philopatric females. The reproductive output resulting from a mating with an immigrant is $b_{0}$ and from a mating with a philopatric female it is $b_{t}$. The difference between $b_{0}$ and $b_{t}$ is the effect of inbreeding depression.

\section{Effect of using a harem mating system}

If we are to change the model of Perrin and Mazalov (2000) to allow only one male to mate with all females in a patch ( $\mathrm{H}$ scenario), for the corresponding male fitness $\hat{W}_{y}$, the notion of Equation A.2 must be adjusted accordingly. The surviving disperser competes with $N b(\tilde{y}+y \tilde{c})$ other males for winning the harem, wins it with probability $1 /(N b(\tilde{y}+y \tilde{c}))$, and then produces $N b_{0}$ offspring. Integrating this into a functional relation analog to Equation A.2 directly shows that this actually does not alter the fitness-defining equation:

$$
\hat{W}_{y}=y_{i j} \tilde{c} \frac{1}{N b(\tilde{y}+y \tilde{c})}\left(N b_{0}\right)+\tilde{y}_{i j} \frac{1}{N b\left(\tilde{y}_{j}+y \tilde{c}\right)}\left(N\left(k_{x} b_{t}+\tilde{k}_{x} b_{0}\right)\right)=W_{y}
$$

Hence, the fitness formulas for males and females are unaffected by the change towards a harem mating scheme, so that also the fitness derivatives and selection gradients remain equal. However, the expected relatedness in such a mating scheme increases greatly - but it increases as much for males as for females. Therefore, we can deduce the evolution of a likewise unbiased - yet highly increased - mean emigration probability. 


\section{References}

Anseeuw, D., Maes, G., Busselen, P., Knapen, D., Snoeks, J., Verheyen, E., Dec. 2008. Subtle population structure and male-biased dispersal in two Copadichromis species (Teleostei, Cichlidae) from Lake Malawi, East Africa. Hydrobiologia 615 (1), 69-79.

Baguette, M., Vansteenwegen, C., Convi, I., Neve, G., 1998. Sex-biased densitydependent migration in a metapopulation of the butterfly Proclossiana eunomia. Acta Oecologica - International Journal Of Ecology 19 (1), 17-24.

Beirinckx, K., Gossum, H. V., Lajeunesse, M. J., Forbes, M. R., 2006. Sex biases in dispersal and philopatry: insights from a meta-analysis based on capture-mark-recapture studies of damselflies. Oikos 113 (3), 539-547.

Bowler, D. E., Benton, T. G., 2005. Causes and consequences of animal dispersal strategies: relating individual behaviour to spatial dynamics. Biological Reviews of the Cambridge Philosophical Society (London) 80 (2), 205-225.

Cano, J. M., Mäkinen, H. S., Merilä, J., 2008. Genetic evidence for male-biased dispersal in the three-spined stickleback (Gasterosteus aculeatus). Molecular Ecology 17 (14), 3234-3242.

Chave, J., 2004. Neutral theory and community ecology. Ecology Letters 7 (3), 241-253.

Clarke, A. L., Saether, B. E., Roskaft, E., 1997. Sex biases in avian dispersal: A reappraisal. Oikos 79 (3), 429-438.

Clobert, J., Danchin, E., Dhont, A. A., Nichols, J. D. (Eds.), 2001. Dispersal. Oxford University Press, New York.

Clobert, J., Le Galliard, J.-F., Cote, J., Meylan, S., Massot, M., 2009. Informed dispersal, heterogeneity in animal dispersal syndromes and the dynamics of spatially structured populations. Ecology Letters 12, 197-209.

Comins, H. N., Hamilton, W. D., May, R. M., 1980. Evolutionarily stable dispersal strategies. Journal of Theoretical Biology 82, 205-230.

Cressman, R., Krivan, V., 2006. Migration dynamics for the ideal free distribution. American Naturalist 168 (3), 384-397.

Dobson, F. S., 1982. Competition for mates and predominant juvenile male dispersal in mammals. Animal Behaviour 30, 1183-1192.

Dubey, S., Brown, G. P., Madsen, T., Shine, R., 2008. Male-biased dispersal in a tropical Australian snake (Stegonotus cucullatua, Colubridae). Molecular Ecology 17 (15), 3506-3514.

Fretwell, S. D., Lucas Jr., H. L., 1970. On territorial behavior and other factors influencing habitat distribution in birds. Acta Biotheoretica 14, 16-36.

Gandon, S., Oct. 1999. Kin competition, the cost of inbreeding and the evolution of dispersal. Journal of Theoretical Biology 200 (4), 345-364.

Gandon, S., Rousset, F., 1999. Evolution of stepping-stone dispersal rates. Proceedings of the Royal Society B: Biological Sciences 266, 2507-2503.

Greenwood, P. J., 1980. Mating systems, philopatry and dispersal in birds and mammals. Animal Behaviour 28 (4), 1140-1162.

Greenwood, P. J., 1983. Mating systems and the evolutionary consequences of dispersal. In: Swingland, I. R., Greenwood, P. J. (Eds.), The ecology of animal movement. Clarendon, pp. 116-131. 
Greenwood, P. J., Harvey, P. H., 1982. The natal and breeding dispersal of birds. Annual Review of Ecology and Systematics 13 (1), 1-21.

Gros, A., Hovestadt, T., Poethke, H. J., 2008. Evolution of sex-biased dispersal: the role of sex-specific dispersal costs, demographic stochasticity, and inbreeding. Ecological Modelling 219, 226-233.

Hammond, R. L., Handley, L. J. L., Winney, B. J., Bruford, M. W., Perrin, N., 2006. Genetic evidence for female-biased dispersal and gene flow in a polygynous primate. Proceedings of the Royal Society B: Biological Sciences 273 (1585), 479-484.

Hanski, I., Gaggiotti, O. E., 2004. Ecology, genetics, and evolution of metapopulations. Elsevier Academic Press, Amsterdam, Boston, Heidelberg.

Hastings, A., 1983. Can spatial variation alone lead to selection for dispersal? Theoretical Population Biology 24, 244-251.

Hirota, T., 2004. The evolution of sex-biased dispersal by pre-dispersal copulation and fluctuating environment. Journal of Animal Ecology 73 (6), 11151120 .

Hirota, T., 2005. The effect of female polyandry and sperm precedence on the evolution of sexual difference in dispersal timing. Journal of Evolutionary Biology 18 (6), 1395-1402.

Keogh, J. S., Webb, J. K., Shine, R., Feb. 2007. Spatial genetic analysis and long-term mark-recapture data demonstrate male-biased dispersal in a snake. Biology Letters 3 (1), 33-35.

Lawson Handley, L. J., Perrin, N., 2007. Advances in our understanding of mammalian sex-biased dispersal. Molecular Ecology 16 (8), 1559-1578.

Leturque, H., Rousset, F., 2003. Joint evolution of sex ratio and dispersal: conditions for higher dispersal rates from good habitats. Evolutionary Ecology $17(1), 67-84$.

Leturque, H., Rousset, F., 2004. Intersexual competition as an explanation for sex-ratio and dispersal biases in polygynous species. Evolution 58 (11), 2398-2408.

Liberg, O., von Schantz, T., 1985. Sex-biased philopatry and dispersal in birds and mammals - the oedipus hypothesis. American Naturalist 126 (1), 129 135 .

McPeek, M. A., Holt, R. D., Dec. 1992. The evolution of dispersal in spatially and temporally varying environments. American Naturalist 140 (6), 10101027.

Metz, J. A., Gyllenberg, M., 2001. How should we define fitness in structured metapopulation models? Including an application to the calculation of evolutionarily stable dispersal strategies. Proceedings of the Royal Society B: Biological Sciences 268 (1466), 499-508.

Möller, L. M., Beheregaray, L. B., 2004. Genetic evidence for sex-biased dispersal in resident bottlenose dolphins (Tursiops aduncus). Molecular Ecology 13 (6), 1607-1612.

Nomura, T., 2005. Effective population size under random mating with a finite number of matings. Genetics 171 (3), 1441-1442.

Öckinger, E., Smith, H. G., 2007. Asymmetric dispersal and survival indicate population sources for grassland butterflies in agricultural landscapes. Ecog- 
raphy 30 (2), 288-298.

Perrin, N., Mazalov, V., 1999. Dispersal and inbreeding avoidance. American Naturalist 154, 282-292.

Perrin, N., Mazalov, V., 2000. Local competition, inbreeding, and the evolution of sex-biased dispersal. American Naturalist 155 (1), 116-127.

Poethke, H. J., Hovestadt, T., 2002. Evolution of density- and patch-sizedependent dispersal rates. Proceedings of the Royal Society B: Biological Sciences 269 (1491), 637-645.

Poethke, H. J., Hovestadt, T., Mitesser, O., 2003. Local extinction and the evolution of dispersal rates: causes and correlations. American Naturalist 161 (4), 631-640.

Poethke, H. J., Pfenning, B., Hovestadt, T., 2007. The relative contributions of individual- and kin-selection in the evolution of density-dependent dispersal rates. Evolutionary Ecology Research 9, 41-50.

Pusey, A. E., 1987. Sex-biased dispersal and inbreeding avoidance in birds and mammals. Trends in Ecology \& Evolution 2 (10), 295-299.

Ronce, O., 2007. How does it feel to be like a rolling stone? Ten questions about dispersal evolution. Annual Review of Ecology, Evolution, and Systematics 38 (1), 231-253.

Sundström, L., Keller, L., Chapuisat, M., 2003. Inbreeding and sex-biased gene flow in the ant Formica exsecta. Evolution 57 (7), 1552-1561.

Taylor, P. D., 1988. An inclusive fitness model for dispersal of offspring. Journal of Theoretical Biology 130 (3), 363-378.

Ujvari, B., Dowton, M., Madsen, T., 2008. Population genetic structure, gene flow and sex-biased dispersal in frillneck lizards (Chlamydosaurus kingii). Molecular Ecology 17 (15), 3557-3564.

Wiener, P., Tuljapurkar, S., Jan. 1994. Migration in variable environments: Exploring life-history evolution using structured population models. Journal of Theoretical Biology 166 (1), 75-90.

Wild, G., Taylor, P. D., 2004. Kin selection models for the co-evolution of the sex ratio and sex-specific dispersal. Evolutionary Ecology Research 6 (4), 481-502.

Williams, D., Rabenold, K. N., 2005. Male-biased dispersal, female philopatry, and routes to fitness in a social corvid. Journal of Animal Ecology 74 (1), 150-159.

Wright, S., 1931. Evolution in mendelian populations. Genetics 16 (2), 97-159. 